International Journal of Trend in Scientific Research and Development (IJTSRD)

Volume: 3 | Issue: 4 | May-Jun 2019 Available Online: www.ijtsrd.com e-ISSN: 2456 - 6470

\title{
Steering Control Adaptive Headlight using Sensor
}

\author{
Mayank Bhardwaj, Kunal Giri, Digvijay Rajput, Mohd. Samsh
}

Department of Mechanical Engineering, ABES Engineering College, Ghaziabad, Uttar Pradesh, India

How to cite this paper: Mayank Bhardwaj | Kunal Giri | Digvijay Rajput | Mohd. Samsh "Steering Control Adaptive Headlight using Sensor" Published in International Journal of Trend in Scientific Research and Development (ijtsrd), ISSN: 24566470, Volume-3 | Issue-4, June 2019, pp.603-604, URL: https://www.ijtsrd.c om/papers/ijtsrd23 872.pdf

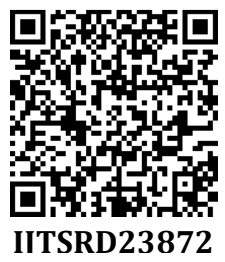

Copyright (C) 2019 by author(s) and International Journal of Trend in Scientific Research and Development Journal. This is an Open Access article distributed under the terms of the Creative Commons

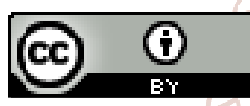

Attribution License (CC BY 4.0) (http://creativecommons.org/licenses/ by/4.0)

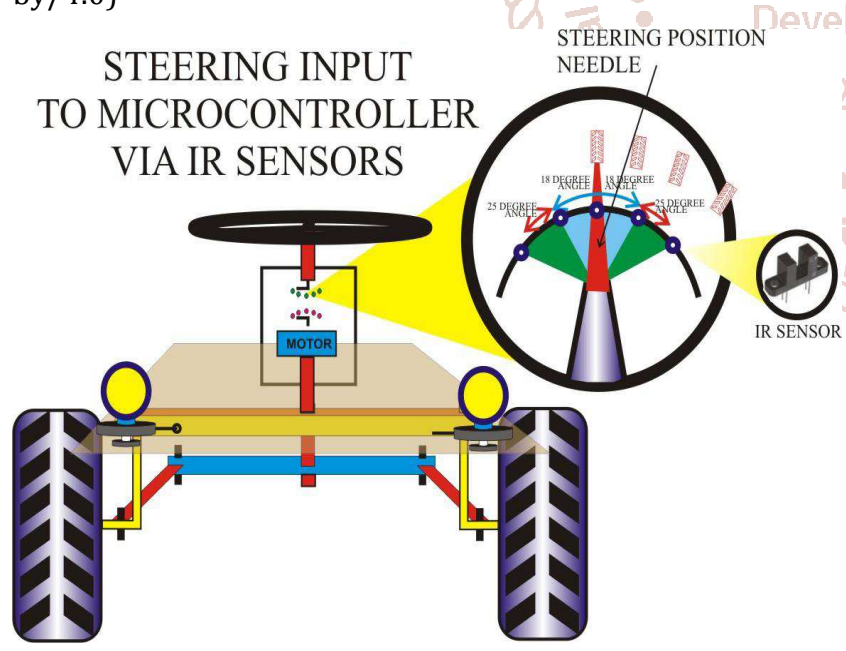

COMPONENTS USED

\section{Microcontrollers}

Microcontroller is a semiconductor component designed by using VLSI(Very Large Scale Integration) technology and contains ALU, CU and registers of a CPU in a single package to perform specific purpose applications.

2. IR INTERRUPTER SENSOR THIS IR INTERRUPTER SENSOR WORK WHEN SOME OBSTRUCTION CUT TRANSMITTER (TX) AND RECEIVER (RX)

\section{ABSTRACT}

Nowadays, road safety is one of the priority of the government to avoid accidents. Road safety adopts those methods that can prevent road users from being seriously injured or killed. Most of the accidents happened during night time when the driver is unable to see its path clearly especially during blind cuts on roads and on hilly areas.

In the conventional headlight's beam of light is focussed only in a single direction that is only in front direction. Our present invention provides a more degree of freedom to the front light which enables these lights to synchronize with the rotation of steering using infrared sensor controlled by microcontroller.

\section{INTRODUCTION}

Conventional headlight follows the tangential path when a vehicle is turned due to which a part of the road remains in dark pose's probability of danger during sharp turn. So, traditional steering mechanism is not connected to the front headlight which makes it one of the major drawbacks of the conventional steering mechanism

In our paper we are focussed on Directional Headlights which improves lightning especially for cornering. The front lights are connected to a link which is connected to the DC motor further such that when the motor rotates the front lights also rotate in the same manner. Motor is connected to the microcontroller which gives input current to the motor to rotate the motor. 


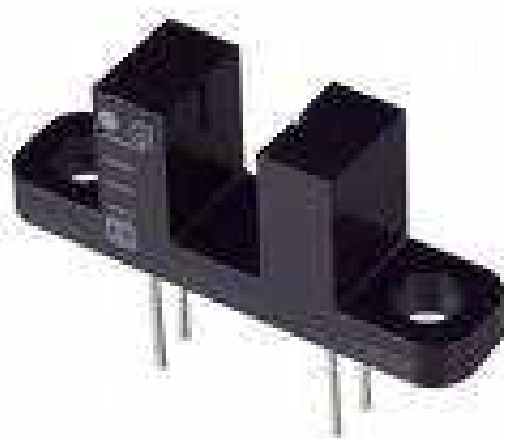

IR INTERRUPTER SENSOR

\section{DC motors}

Powerful gear motor. 10 RPM @ 12 Vdc / 90 mA (no-load). Operates on 4-15 Vdc. 3.6" x 2.36" x 2.24" overall dimensions. DC motor and final drive shaft both extend from same side of plastic gearbox. 5/16" diameter flatted shaft is 0.9 " long. 8" pigtail leads.

\section{TRANSISTOR}

Transistor is a device which consist of base, collector and emitter region in which emitter is heavily doped, collector is medium doped and base is lightly doped. Transistor can be used as a switch or as an amplifier used to amplify the electrical signal. In our project transistor is used as a switch used to on and off the circuit.

\section{RELAY}

Relay is used to isolate two circuits. Relay isolate high voltage side and the low voltage side such that components remains safe and there is no excess current flow through components.

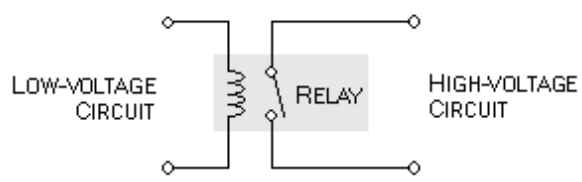

Figure 1: A relay providing isolation between two circuits

\section{DIODE}

The simplest semiconductor device is made up of a sandwich of P-type semi conducting material, with contacts provided to connect the $\mathrm{p}$-and n-type layers to an external circuit. This is a junction Diode. In the forward biasing of the circuit the positive and the negative terminal of the battery are connected to the p-type material and n-type material respectively. Due to this the thickness of the depletion layer decreases which allows large current to flow in the circuit. In the reverse biasing connections are reversed such that positive terminal of battery is connected to the n-type material and negative terminal of the battery is connected to the p-type material respectively. Due to this the thickness of the depletion layer increases which allows very less current to flow through it.

\section{WORKING}

Circuit works on the principle of infrared sensing of signal produced due to movement of steering.

When steering gets rotated and comes in the vicinity of infrared sensor it cuts infrared signal by obstructing signal between transmitter and receiver which generates pulse which gets transmitted to microcontroller.

Microcontroller process signal from IR sensor and generates a current signal for DC motor through relay. After getting signal from microcontroller DC motor starts rotating which in turn rotates head lights.

After intended rotation of head lights by DC motor again an IR signal gets generated by moving mechanism attached to DC motor which gets transmitted to microcontroller which process it further and stops current signal to DC motor and motor gets stopped and we get the desired amount of rotation of head lights

In this way head lights gets synchronized with steering and we get desired result.
STEERING INPUT

TO

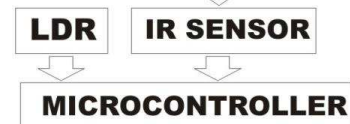

LIGHT IR SENSOR MOTOR SIDE

\section{CALCULATION}

Total angle through which wheels turn is $120^{\circ}$ (i.e from extreme right to extreme left).

Total angle made by steering is $180^{\circ}$

Steering ratio $=180^{\circ} / 120^{\circ}=1.5$

Battery Output $=18 \mathrm{~V}$

Motor 12 V(DC) / $90 \mathrm{~mA}$

\section{CONCLUSION}

This can be very useful system in our car when we are cutting to the car right/lift for more front clear visibility. It provides light to the vehicles in short turning and protect from accident.

\section{REFERENCES}

[1] Japanese Patent Application Laid-Open No. H8-192674.

[2] US Patent No. 6,309,082, Inventor - Tien-Ching Wu.

[3] Automobile engineering by Dr. kirpal singh chapter: front axle steering systems.

[4] The 8051 Microcontroller and Embedded Systems: Using Assembly and C by Muhammad Ali Mazidi, Rolin McKinlay and Janice Gillispie Mazidi. 